\title{
SIMPLE CONDITIONS FOR PRACTICAL STABILITY OF POSITIVE FRACTIONAL DISCRETE-TIME LINEAR SYSTEMS
}

\author{
MikoŁaJ BUSŁOWICZ, TADEUSZ KACZOREK
}

Faculty of Electrical Engineering

Białystok Technical University, ul. Wiejska 45D, 15-351 Białystok, Poland

e-mail: busmiko@pb.edu.pl,kaczorek@isep.pw.edu.pl

\begin{abstract}
In the paper the problem of practical stability of linear positive discrete-time systems of fractional order is addressed. New simple necessary and sufficient conditions for practical stability and for practical stability independent of the length of practical implementation are established. It is shown that practical stability of the system is equivalent to asymptotic stability of the corresponding standard positive discrete-time systems of the same order. The discussion is illustrated with numerical examples.
\end{abstract}

Keywords: linear system, positive, discrete-time, fractional, stability, practical stability.

\section{Introduction}

In positive systems inputs, state variables and outputs take only non-negative values. A variety of models having positive linear behavior can be found in engineering, management science, economics, social sciences, biology and medicine, etc. An overview of state of the art in positive systems theory is given in (Farina and Rinaldi, 2000; Kaczorek, 2002).

In the last decades, the problem of the analysis and synthesis of dynamical systems described by fractional order differential (or difference) equations has been considered in many papers, see, e.g., (Das, 2008; Kilbas et al., 2006; Podlubny, 1999; Sabatier et al., 2007; Sierociuk, 2007; Vinagre et al., 2002).

Stability and robust stability problems of fractional linear systems were investigated in (Busłowicz, 2008a; 2008b; 2008c; 2008d; Chen, 2006; Dzieliński and Sierociuk, 2006; Gałkowski and Kumert, 2005; Gałkowski et al., 2006; Sierociuk, 2007; Vinagre et al., 2002). The reachability and controllability to zero of positive fractional linear systems were considered in (Kaczorek, 2007a; 2007b; 2008a; 2008b). The notion of standard and positive 2D fractional linear systems was introduced in (Kaczorek, 2008c; 2008d).

The concept of practical stability of positive fractional discrete-time systems was introduced and conditions for practical stability were given by Kaczorek (2008e).
In this paper, simple new necessary and sufficient conditions for practical stability of positive fractional discrete-time linear systems will be established.

The following notation will be used: $\mathbb{R}^{n \times m}$ is the set of $n \times m$ real matrices and $\mathbb{R}^{n}=\mathbb{R}^{n \times 1} ; \mathbb{R}_{+}^{n \times m}$ is the set of $n \times m$ real matrices with non-negative entries and $\mathbb{R}_{+}^{n}=$ $\mathbb{R}_{+}^{n \times 1} ; \mathbb{Z}_{+}$is the set of non-negative integers; $I_{n}$ is the $n \times$ $n$ identity matrix; a vector $x$ with positive all entries (resp. with negative all entries) will be called strictly positive and denoted by $x>0$ (resp. strictly negative and denoted by $x<0)$.

\section{Problem formulation}

Let us consider the discrete-time linear system of fractional order $\alpha \in(0,1)$, described by the state equation

$$
\begin{aligned}
& \Delta^{\alpha} x_{i+1}=A x_{i}+B u_{i}, \\
& i \in \mathbb{Z}_{+}, \quad 0<\alpha<1,
\end{aligned}
$$

where $x_{i} \in \mathbb{R}^{n}$ is the state vector, $u_{i} \in \mathbb{R}^{m}$ is the input vector, $A \in \mathbb{R}^{n \times n}, B \in \mathbb{R}^{n \times m}$ and $\Delta^{\alpha} x_{i}$ is the fractional difference defined by (Kaczorek, 2007a)

$$
\Delta^{\alpha} x_{i}=x_{i}+\sum_{j=1}^{i}(-1)^{j}\left(\begin{array}{l}
\alpha \\
j
\end{array}\right) x_{i-j},
$$


$\alpha \in(0,1)$, with

$$
\left(\begin{array}{l}
\alpha \\
j
\end{array}\right)=\frac{\alpha(\alpha-1) \ldots(\alpha-j+1)}{j !}, \quad j>0 .
$$

Using (2a), we may write Eqn. (1) in the form

$$
x_{i+1}=A_{\alpha} x_{i}+\sum_{j=1}^{i} c_{j}(\alpha) x_{i-j}+B u_{i}, \quad i \in \mathbb{Z}_{+},
$$

where

$$
A_{\alpha}=A+I_{n} \alpha,
$$

and

$$
c_{j}=c_{j}(\alpha)=(-1)^{j}\left(\begin{array}{c}
\alpha \\
j+1
\end{array}\right), \quad j=1,2, \ldots .
$$

The fractional system (1) is positive (internally), i.e., $x_{i} \in \mathbb{R}_{+}^{n}$ for all $i \in \mathbb{Z}_{+}$for any initial conditions $x_{0} \in \mathbb{R}_{+}^{n}$ and all input sequences $u_{i} \in \mathbb{R}_{+}^{m}, i \in \mathbb{Z}_{+}$, if and only if (Kaczorek, 2007a)

$$
A_{\alpha}=A+I_{n} \alpha \in \mathbb{R}_{+}^{n \times n}, \quad B \in \mathbb{R}_{+}^{n \times m} .
$$

The system (3) has a variable number of delays. For any discrete time $i=N$, the number of delays is equal to $N$ and this number increases to infinity if $i \rightarrow \infty$.

From (5) and (2b) it follows that $c_{j}=c_{j}(\alpha)>0$ for $\alpha \in(0,1)$ and $j=1,2, \ldots$ Moreover, the coefficients $c_{j}=c_{j}(\alpha)$ strongly decrease for increasing $j$. Therefore, in practical problems it is assumed that $j$ is bounded by some natural number $h$. This number is called the length of the practical implementation. In this case, Eqn. (3) takes the form

$$
x_{i+1}=A_{\alpha} x_{i}+\sum_{j=1}^{h} c_{j}(\alpha) x_{i-j}+B u_{i}, \quad i \in \mathbb{Z}_{+},
$$

with the initial conditions $x_{-i} \in \mathbb{R}_{+}^{n}, i=0,1, \ldots, h$. Equation (7) describes a linear discrete-time system with $h$ delays in state.

The time-delay system (7) will be called a practical implementation of the fractional system (1).

Definition 1. (Kaczorek, 2007e) The positive fractional system (3) is called practically stable if and only if the system (7) is asymptotically stable.

The positive linear system without delays, equivalent to (7), has the form

$$
\tilde{x}_{i+1}=\tilde{A} \tilde{x}_{i}+\tilde{B} u_{i}, \quad i \in \mathbb{Z}_{+},
$$

where $\tilde{x}_{i}=\left[x_{i}^{\mathrm{T}}, x_{i-1}^{\mathrm{T}}, \cdots, x_{i-h}^{\mathrm{T}}\right]^{\mathrm{T}} \in \mathbb{R}_{+}^{\tilde{n}}, \tilde{A} \in \mathbb{R}_{+}^{\tilde{n} \times \tilde{n}}$, $\tilde{B} \in \mathbb{R}_{+}^{\tilde{n} \times m}, \tilde{n}=(h+1) n$ and

$$
\tilde{A}=\left[\begin{array}{cccccc}
A_{\alpha} & c_{1} I_{n} & c_{2} I_{n} & \cdots & c_{h-1} I_{n} & c_{h} I_{n} \\
I_{n} & 0 & 0 & \cdots & 0 & 0 \\
0 & I_{n} & 0 & \cdots & 0 & 0 \\
\vdots & \vdots & \vdots & \ddots & \vdots & \vdots \\
0 & 0 & 0 & \cdots & I_{n} & 0
\end{array}\right]
$$

$$
\tilde{B}=\left[\begin{array}{c}
B \\
0 \\
0 \\
\vdots \\
0
\end{array}\right]
$$

The practical stability problem was formulated and solved in (Kaczorek, 2008e).

Theorem 1. (Kaczorek, 2008e) The positive fractional system (3) is practically stable if and only if one of the following equivalent conditions holds:

1. Eigenvalues $z_{1}, z_{2}, \ldots, z_{\tilde{n}}$ of the matrix $\tilde{A}$ defined in (9) have moduli less than 1.

2. All coefficients of the characteristic polynomial of the matrix $\tilde{A}-I_{\tilde{n}}$ are positive.

3. All leading principal minors of the matrix $I_{\tilde{n}}-\tilde{A}$ are positive.

4. $\rho(\tilde{A})<1$, where

$$
\rho(\tilde{A})=\max _{1 \leq k \leq \tilde{n}}\left|z_{k}\right|
$$

is the spectral radius of the matrix $\tilde{A}$.

5. There exist strictly positive vectors $\bar{x}_{i} \in \mathbb{R}_{+}^{n}, i=$ $0,1, \cdots, h$, satisfying the conditions $\bar{x}_{0}<\bar{x}_{1}, \bar{x}_{1}<$ $\bar{x}_{2}, \ldots, \bar{x}_{h-1}<\bar{x}_{h}$, such that $A_{\alpha} \bar{x}_{0}+c_{1} \bar{x}_{1}+\cdots+$ $c_{h} \bar{x}_{h}<\bar{x}_{h}$.

Note that the size of the matrix $\tilde{A} \in \mathbb{R}^{\tilde{n} \times \tilde{n}}$ with $\tilde{n}=$ $(h+1) n$, of the form defined in (9), depends on the length $h$ of practical implementation. Therefore, the application of Theorem 1 to practical stability analysis of the positive fractional system (3) is a difficult problem for large $h$.

The aim of the paper is to give new simple necessary and sufficient conditions for practical stability of the positive fractional system (3). We show that practical stability of this system with a given length $h$ of practical implementation is equivalent to asymptotic stability of the corresponding positive system without delays of the same order as the system (3), i.e., of order extremely less than the order of the system (8) (equal to $\tilde{n}=(h+1) n$ ).

\section{Solution of the problem}

From the structure of the matrix $\tilde{A} \in \mathbb{R}_{+}^{\tilde{n} \times \tilde{n}}$ of the form given in (9) and from the condition (3) of Theorem 1 we have the following important lemma.

Lemma 1. If the positive fractional system (3) is practically stable with the given length $h=H$ of practical implementation, then this system is also practically stable for any positive $h<H$. 
Theorem 2. The positive fractional system (3) is practically stable if and only if there exists a strictly positive vector $\lambda \in \mathbb{R}_{+}^{n}$ such that

$$
\left[A_{\alpha}+\sum_{j=1}^{h} c_{j} I_{n}-I_{n}\right] \lambda<0, \quad \lambda>0 .
$$

Proof. We show that (10) is necessary and sufficient for asymptotic stability of the positive time-delay system (7). Necessity. Let $X \in \mathbb{R}_{+}^{n \times n}$ have all eigenvalues with absolute values less than one. It is easy to show that

$$
\left[I_{n}-X\right]^{-1}=\sum_{k=1}^{\infty} X^{k} \in \mathbb{R}_{+}^{n \times n} .
$$

Hence, if the positive system (7) is asymptotically stable, then from (11) we have $\left[I_{n}-A_{\alpha}-\sum_{j=1}^{h} c_{j} I_{n}\right]^{-1} \in \mathbb{R}_{+}^{n \times n}$ and

$$
\gamma=\left[I_{n}-A_{\alpha}-\sum_{j=1}^{h} c_{j} I_{n}\right]^{-1} 1_{n}>0
$$

where $1_{n}=\left[\begin{array}{lll}1 & \cdots & 1\end{array}\right]^{\mathrm{T}}$.

From the above it follows that if the system (7) is asymptotically stable, then the condition (10) holds for $\lambda=\gamma>0$.

Sufficiency. Let us consider the dual system (with $B \equiv 0$ )

$$
x_{i+1}=A_{\alpha}^{\mathrm{T}} x_{i}+\sum_{j=1}^{h} c_{j}(\alpha) x_{i-j}, \quad i \in \mathbb{Z}_{+},
$$

which is positive and asymptotically stable if and only if the original system (7) is positive and asymptotically stable.

As a Lyapunov function for the dual system (13), we may choose the following function:

$$
V\left(x_{i}\right)=x_{i}^{\mathrm{T}} \lambda+\sum_{j=1}^{h} c_{j} \sum_{r=i-j}^{i-1} x_{r}^{\mathrm{T}} \lambda, \quad \lambda \in \mathbb{R}_{+}^{n},
$$

which is positive for non-zero $x_{i} \in \mathbb{R}_{+}^{m}$ and for strictly positive $\lambda$.

From (14) and (13), we have

$$
\begin{aligned}
\Delta V\left(x_{i}\right)= & V\left(x_{i+1}\right)-V\left(x_{i}\right) \\
= & x_{i+1}^{\mathrm{T}} \lambda+\sum_{j=1}^{h} c_{j} \sum_{r=i+1-j}^{i} x_{r}^{\mathrm{T}} \lambda-x_{i}^{\mathrm{T}} \lambda \\
& -\sum_{j=1}^{h} c_{j} \sum_{r=i-j}^{i-1} x_{r}^{\mathrm{T}} \lambda \\
= & x_{i}^{\mathrm{T}}\left[A_{\alpha}+\sum_{j=1}^{h} c_{j} I_{n}-I_{n}\right] \lambda<0 .
\end{aligned}
$$

Hence, the condition (10) implies $\Delta V\left(x_{i}\right)<0$ and the positive system (7) is asymptotically stable. This means that the fractional system (3) is practically stable.
Theorem 3. The positive fractional system (3) is practically stable if and only if the standard positive system

$$
x_{i+1}=D x_{i}, \quad i \in \mathbb{Z}_{+},
$$

with

$$
D=A_{\alpha}+\sum_{j=1}^{h} c_{j} I_{n} \in \mathbb{R}_{+}^{n \times n},
$$

is asymptotically stable.

Proof. In (Kaczorek, 2007c), it was shown that the positive system (15) is asymptotically stable if and only if there exists a strictly positive vector $\lambda \in \mathbb{R}_{+}^{n}$ such that $\left[D-I_{n}\right] \lambda<0$. Hence, the proof follows directly from Theorem 2 and (16).

The application of Theorem 3 requires computing of the coefficients $c_{j}=c_{j}(\alpha)(j=1,2, \ldots, h)$ defined by (5) and (2b). These coefficients can be easily computed by the following simple algorithm suitable for computer programming:

$$
c_{j+1}=c_{j} \frac{j+1-\alpha}{j+2}, \quad j=1,2, \ldots, h,
$$

where $c_{1}=0.5 \alpha(1-\alpha)$.

From Theorems 2 and 3 and the stability condition of the positive system (15), given in (Farina and Rinaldi, 2000; Kaczorek, 2002; 2007c; 2008e), we have the following theorem and lemma.

Theorem 4. The positive fractional system (3) is practically stable if and only if one of the following equivalent conditions holds:

1. The eigenvalues $z_{1}, z_{2}, \ldots, z_{n}$ of the matrix $D$ defined by (16) have moduli less than 1 .

2. All leading principal minors of the matrix $I_{n}-D$ are positive.

3. All coefficients of the characteristic polynomial of the matrix $D-I_{n}$ are positive.

4. $\rho(D)<1$, where

$$
\rho(D)=\max _{1 \leq k \leq n}\left|z_{k}\right|
$$

is the spectral radius of the matrix $D$.

Lemma 2. The positive fractional system (3) is practically unstable if one of the following equivalent conditions holds:

1. At least one diagonal entry of the matrix $D$ defined by (16) is greater than 1.

2. The positive system $x_{i+1}=A_{\alpha} x_{i}$ ( $A_{\alpha}$ is defined by (4)) is unstable. 
3. At least one diagonal entry of the matrix $A_{\alpha}$ is greater than 1.

Now we consider the problem of practical stability of the positive system (3) in the case when $h \rightarrow \infty$ in (7).

From Definition 1 for $h \rightarrow \infty$ and Lemma 1 we have the following definition.

Definition 2. The positive fractional system (3) with fixed $\alpha \in(0,1)$ is called practically stable independent of the length of practical implementation if and only if the positive system (7) is asymptotically stable for all $h \geq 1$.

First, we prove the following important lemma.

Lemma 3. The following equality holds:

$$
\sum_{j=1}^{\infty} c_{j}=1-\alpha
$$

Proof. In (Sierociuk, 2007), it was shown that

$$
\sum_{j=0}^{\infty}(-1)^{j}\left(\begin{array}{l}
\alpha \\
j
\end{array}\right)=0
$$

It easy to see that

$$
\sum_{j=0}^{\infty}(-1)^{j}\left(\begin{array}{c}
\alpha \\
j
\end{array}\right)=1-\alpha-\sum_{j=1}^{\infty}(-1)^{j}\left(\begin{array}{c}
\alpha \\
j+1
\end{array}\right) .
$$

Taking into account (19), (20) and (5), we obtain (18).

From the above and Theorem 3 , for $h \rightarrow \infty$ one obtains the following conditions for practical stability independent of the length of practical implementation.

Theorem 5. The positive fractional system (3) is stable independent of the length of practical implementation if and only if the standard positive system

$$
x_{i+1}=D_{\infty} x_{i}, \quad i \in \mathbb{Z}_{+},
$$

is asymptotically stable, where $D_{\infty}=A+I_{n} \in \mathbb{R}_{+}^{n \times n}$.

Proof. From (4), (18) and (16), for $h \rightarrow \infty$ we have

$$
\begin{aligned}
D & =A_{\alpha}+\sum_{j=1}^{\infty} c_{j} I_{n} \\
& =A+I_{n} \alpha+I_{n}(1-\alpha)=A+I_{n} .
\end{aligned}
$$

Hence, the proof follows directly from Theorem 3.
From Theorem 5 we have the following important corollary.

Corollary 1. If the positive fractional system (3) if practically stable independent of the length of practical implementation, then it is practically stable for all $h \geq 1$ and for any $\alpha \in(0,1)$.

Applying the well known stability conditions to the positive system (21), one obtains the following theorem and lemma.

Theorem 6. The positive fractional system (3) is practically stable independent of the length of practical implementation if and only if one of the following equivalent conditions holds:

1. The eigenvalues $z_{1}, z_{2}, \cdots, z_{n}$ of the matrix $D_{\infty}=$ $A+I_{n}$ have moduli less than 1 .

2. All leading principal minors of the matrix $-A$ are positive.

3. All coefficients of the characteristic polynomial of the matrix $A$ are positive.

4. $\rho\left(D_{\infty}\right)<1$, where

$$
\rho\left(D_{\infty}\right)=\max _{1 \leq k \leq n}\left|z_{k}\right|
$$

is the spectral radius of the matrix $D_{\infty}$.

Lemma 4. The positive fractional system (3) is not practically stable independent of the length of practical implementation if at least one diagonal entry of the matrix $A$ is positive.

Proof. If at least one diagonal entry of the matrix $A$ is positive, then at least one diagonal entry of the matrix $D_{\infty}=A+I_{n}$ is greater than 1 and the system (21) is unstable. This means that the positive fractional system (3) is not practically stable independent of the length of practical implementation.

Let us consider the positive scalar discrete-time system of fractional order $\alpha \in(0,1)$ described by the state equation (1), where $A=a$ and $B=b$ are real numbers such that $a_{\alpha}=a+\alpha \geq 0, b \geq 0$. In this case, from (3) we have

$$
x_{i+1}=a_{\alpha} x_{i}+\sum_{j=1}^{i} c_{j}(\alpha) x_{i-j}+b u_{i}, \quad i \in \mathbb{Z}_{+} .
$$

Practical implementation of (23) with the given length $h$ of practical implementation has the form

$$
x_{i+1}=a_{\alpha} x_{i}+\sum_{j=1}^{h} c_{j}(\alpha) x_{i-j}+b u_{i}, \quad i \in \mathbb{Z}_{+},
$$

with the initial conditions $x_{-i} \in \mathbb{R}_{+}^{n}, i=0,1, \ldots, h$. 
Lemma 5. (Busłowicz, 2008d) If $\alpha \in(0,1)$, then $\delta_{h}(\alpha) \in(0,1)$, where

$$
\delta_{h}(\alpha)=1-\alpha-\sum_{j=1}^{h} c_{j}(\alpha) .
$$

From Theorems 4 and 6 and Lemma 2, we have the following theorem (see also (Busłowicz, 2008d)).

Theorem 7. The positive scalar discrete-time system (23) of fractional order $\alpha \in(0,1)$ is

1. practically stable with the given length $h \geq 1$ of practical implementation if and only if $a<\delta_{h}(\alpha)$,

2. practically stable independent of the length of practical implementation if and only if $0>a \geq-\alpha$,

3. practically unstable independent of the length of practical implementation if $a>1-\alpha$.

\section{Illustrative examples}

Example 1. Consider the positive fractional order linear system described by the equation

$$
\begin{array}{r}
\Delta^{\alpha} x_{i+1}=\left[\begin{array}{cc}
-0.3 & 0.2 \\
0.2 & a
\end{array}\right] x_{i}, \\
\quad \alpha=0.3, \quad a \in \mathbb{R} .
\end{array}
$$

We wish to check the practical stability of the system with the length of practical implementation $h=100$.

From (6), it follows that the system is positive if and only if

$$
A_{\alpha}=A+I_{2} \alpha=\left[\begin{array}{cc}
0 & 0.2 \\
0.2 & 0.3+a
\end{array}\right] \in \mathbb{R}_{+}^{2 \times 2},
$$

that is, $a \geq-0.3$.

Computing the coefficients $c_{j}(j=1,2, \ldots, h=$ $100)$ from (17) and the matrix $D$ from (16), one obtains

$$
\sum_{j=1}^{100} c_{j}=0.5073
$$

and

$$
D=\left[\begin{array}{cc}
0.5073 & 0.2 \\
0.2 & a+0.8073
\end{array}\right]
$$

The leading principal minors of the matrix $I_{2}-D$ are as follows: $\Delta_{1}=0.4927, \Delta_{2}=-0.4927 a+0.0549$. They are positive for $a<0.1114$. Hence, according to the condition 2 of Theorem 4, the fractional system (26) with $h=100$ is practically stable if and only if $-0.3 \leq a<$ 0.1114 .
Note that the application of Theorem 1 to practical stability analysis of the positive system (26) requires checking asymptotic stability of the matrix $\tilde{A} \in \mathbb{R}^{\tilde{n} \times \tilde{n}}$ (defined in (9)) with $\tilde{n}=(h+1) n=202$.

Example 2. Check practical stability independent of the length of practical implementation of the positive fractional order linear system described by (1) with $\alpha=0.3$ and

$$
A=\left[\begin{array}{ccc}
a & 0.3 & 0 \\
0.2 & b & 0.1 \\
0 & 0.2 & c
\end{array}\right], \quad a, b, c \in \mathbb{R} .
$$

The system (21) is positive if and only if $D_{\infty}=A+$ $I_{3} \in \mathbb{R}_{+}^{3 \times 3}$, i.e., $a \geq-1, b \geq-1$ and $c \geq-1$.

Computing the leading principal minors of the matrix $-A$ one obtains $\Delta_{1}=-a, \Delta_{2}=a b-0.06, \Delta_{3}=$ $\operatorname{det}(-A)=-a b c+0.02 a+0.06 c$. They are positive if and only if $a<0 ; a b<0.06 ; c(a b-0.06)<0.02 a$.

Hence, the fractional system is positive and practically stable independent of the length of practical implementation (according to the condition 2 of Theorem 6) if and only if

$$
\begin{gathered}
-1 \leq a<0, \quad-1 \leq b<\frac{0.06}{a} \\
-1 \leq c<\frac{0.02 a}{a b-0.06} .
\end{gathered}
$$

It is easy to check that inequalities (28) hold for, e.g., $a=-0.5 ; b=-0.2$ and $c=-0.3$. This means that the positive system (1) with $\alpha=0.3$ and the state matrix (27) with $a=-0.5 ; b=-0.2$ and $c=-0.3$ is practically stable independent of the length of practical implementation.

\section{Concluding remarks}

The practical stability problem of the positive discretetime system (3) of fractional order $0<\alpha<1$ has been addressed. New simple necessary and sufficient conditions for practical stability and for practical stability independent of the length of practical implementation were established (Theorems 3, 4 and 5, 6, respectively).

It was shown that the positive system (3) is

(i) practically stable with the given length of practical implementation if and only if the positive standard discrete-time system (15) is asymptotically stable,

(ii) practically stable independent of the length of practical implementation if and only if the positive standard discrete-time system (21) is asymptotically stable.

In the case of the scalar positive discrete-time fractional order system (23), the stability conditions were given in Theorem 7 . 


\section{Acknowledgment}

This work was supported by the Ministry of Science and Higher Education in Poland under Grant No. N N514 193933.

\section{References}

Busłowicz, M. (2008a). Stability of linear continuous-time fractional systems of commensurate order, Pomiary, Automatyka, Robotyka: 475-484, (on CD-ROM, in Polish); Journal of Automation, Mobile Robotics and Intelligent Systems 3(1): 16-21 .

Busłowicz, M. (2008b). Frequency domain method for stability analysis of linear continuous-time fractional systems, in K. Malinowski and L. Rutkowski (Eds.), Recent Advances in Control and Automation, Academic Publishing House EXIT, Warsaw, pp. 83-92.

Busłowicz, M. (2008c). Robust stability of convex combination of two fractional degree characteristic polynomials, Acta Mechanica et Automatica 2(2): 5-10.

Busłowicz, M. (2008d). Practical robust stability of positive fractional scalar discrete-time systems, Zeszyty Naukowe Politechniki Ślqskiej: Automatyka 151: 25-30, (in Polish).

Chen, Y.-Q., Ahn, H.-S. and Podlubny, I. (2006). Robust stability check of fractional order linear time invariant systems with interval uncertainties, Signal Processing 86(10): 2611-2618.

Das, S. (2008). Functional Fractional Calculus for System Identification and Controls, Springer, Berlin.

Dzieliński, A. and Sierociuk, D. (2006). Stability of discrete fractional state-space systems, Proceedings of the 2-nd IFAC Workshop on Fractional Differentiation and Its Applications, IFAC FDA'06, Porto, Portugal, pp. 518-523.

Farina, L. and Rinaldi, S. (2000). Positive Linear Systems: Theory and Applications, J. Wiley, New York, NY.

Gałkowsk,i K. and Kummert, A. (2005). Fractional polynomials and $\mathrm{nD}$ systems, Proceedings of the IEEE International Symposium on Circuits and Systems, ISCAS'2005, Kobe, Japan, (on CD-ROM).

Gałkowski, K., Bachelier, O. and Kummert, A. (2006). Fractional polynomial and $\mathrm{nD}$ systems-A continuous case, Proceedings ot the IEEE Conference on Decision and Control, San Diego, CA, USA, pp. 2913-2917.

Kaczorek, T. (2002). Positive $1 D$ and $2 D$ Systems, SpringerVerlag, London.

Kaczorek, T. (2007a). Reachability and controllability to zero of positive fractional discrete-time systems, Machine Intelligence and Robotic Control 6(4): 139-143.

Kaczorek, T. (2007b). Reachability and controllability to zero of cone fractional linear systems, Archives of Control Sciences 17(3): 357-367.

Kaczorek, T. (2007c). Choice of the forms of Lyapunov functions for positive 2D Roesser model, International Journal of Applied Mathematics and Computer Science 17(4): 471-475.

Kaczorek, T. (2008a). Fractional positive continuous-time linear systems and their reachability, International Journal of Applied Mathematics and Computer Science 18(2): 223-228.
Kaczorek, T. (2008b). Reachability and controllability to zero tests for standard and positive fractional discrete-time systems, Journal of Automation and System Engineering 42(6-7-8): 769-787.

Kaczorek, T. (2008c). Fractional 2D linear systems, Journal of Automation, Mobile Robotics and Intelligent Systems 2(2): 5-9.

Kaczorek, T. (2008d). Positive different orders fractional 2D linear systems, Acta Mechanica et Automatica 2(2): 51-58.

Kaczorek, T. (2008e). Practical stability of positive fractional discrete-time systems, Bulletin of the Polish Academy of Sciences: Technical Sciences 56(4): 313-317.

Kilbas, A. A., Srivastava, H. M. and Trujillo, J. J. (2006). Theory and Applications of Fractional Differential Equations, Elsevier, Amsterdam.

Podlubny, I. (1999). Fractional Differential Equations, Academic Press, San Diego, CA.

Sabatier, J., Agrawal, O. P. and Machado, J. A. T. (Eds.) (2007). Advances in Fractional Calculus, Theoretical Developments and Applications in Physics and Engineering, Springer, London.

Sierociuk, D. (2007). Estimation and Control of Discrete Dynamical Systems of Fractional Order in State Space, Ph.D. thesis, Faculty of Electrical Engineering, Warsaw University of Technology, Warsaw, (in Polish).

Vinagre, B. M., Monje, C. A. and Calderon, A. J. (2002). Fractional order systems and fractional order control actions, Proceedings of the IEEE CDC Conference Tutorial Workshop: Fractional Calculus Applications in Automatic Control and Robotics, Las Vegas, NY, pp. 15-38.

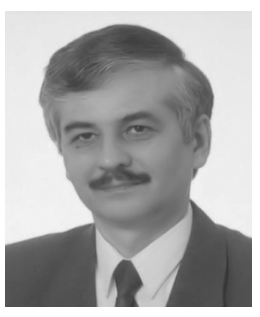

Mikołaj Busłowicz received the M.Sc., Ph.D. and D.Sc. degrees in electrical engineering in 1974, 1977 and 1988, respectively, all from the Faculty of Electrical Engineering of the Warsaw University of Technology. Since 1978 he has been with Białystok Technical University. In the years 1988-1993 and 1993-1999 he was respectively a deputy dean and the dean of the Faculty of Electrical Engineering, and in the period of 1999-2005 he was a deputy rector of the University. In 2002 he became a professor and since 2005 has been a full professor at Białystok Technical University. Currently, he is the head of the Department of Automatics and Electronics. Since 2004 he has been a member of the Committee on Automatic Control and Robotics of the Polish Academy of Sciences. His main research interests include the analysis and synthesis of time delay systems, positive systems, robust stability, and computer-aided design of control systems. He has published three books and about 150 scientific papers.

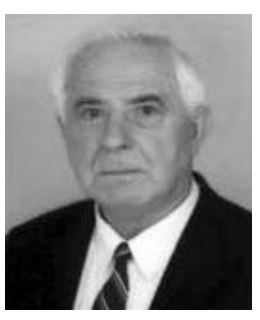

Tadeusz Kaczorek received the M.Sc., Ph.D. and D.Sc. degrees in electrical engineering from the Warsaw University of Technology in 1956, 1962 and 1964, respectively. In the years 1968-69 he was the dean of the Electrical Engineering Faculty, and in the period of 1970-73 he was a deputy rector of the Warsaw University of Technology. In 1971 he became a professor and in 1974 a full professor at the Warsaw University of Technology. Since 2003 he 
has been a professor at Białystok Technical University. In 1986 he was elected a corresponding member and in 1996 a full member of the Polish Academy of Sciences. In the years 1988-1991 he was the director of the Research Centre of the Polish Academy of Sciences in Rome. In May 2004 he was elected an honorary member of the Hungarian Academy of Sciences. He has been granted honorary doctorates by several universities. His research interests cover the theory of systems and automatic control systems theory, especially singular multidimensional systems, positive multidimensional systems, and singular positive 1D and $2 \mathrm{D}$ systems.
He has initiated research in the field of singular 2D and positive 2D systems. He has published 21 books (six in English) and over 850 scientific papers. He has also supervised $67 \mathrm{Ph} . \mathrm{D}$. theses. He is the editor-in-chief of the Bulletin of the Polish Academy of Sciences: Technical Sciences and a member of editorial boards of about ten international journals.

Received: 10 September 2008

Revised: 25 January 2009 